\title{
Meta
}

Journal des traducteurs

Translators' Journal

\section{Percebois, J. (2002) : Terminologie anglais-français du commerce international : théories, politiques, accords et institutions, Paris, Economica, 296 p.}

\section{Jeanne Dancette}

Volume 48, numéro 4, décembre 2003

URI : https://id.erudit.org/iderudit/008742ar

DOI : https://doi.org/10.7202/008742ar

Aller au sommaire du numéro

Éditeur(s)

Les Presses de l'Université de Montréal

ISSN

0026-0452 (imprimé)

1492-1421 (numérique)

Découvrir la revue

Citer ce compte rendu

Dancette, J. (2003). Compte rendu de [Percebois, J. (2002) : Terminologie anglais-français du commerce international : théories, politiques, accords et institutions, Paris, Economica, 296 p.] Meta, 48(4), 627-629.

https://doi.org/10.7202/008742ar d'utilisation que vous pouvez consulter en ligne.

https://apropos.erudit.org/fr/usagers/politique-dutilisation/ 


\section{RÉFÉRENCES}

BAKer, M. (1998) : «Réexplorer la langue de la traduction : une approche par corpus», Meta 434, p. $480-485$.

Eco, U. (1992): Les limites de l'interprétation, Paris, Grasset.

Éveraert-Desmedt, N. (1990): Le processus interprétatif, Mardaga, Liège.

Folkart, B. (1991): Le conflit des énonciations, Candiac (Québec), Balzac.

Klingberg, G. (1986): Children's Fiction in the Hands of the Translators, Studia psychologica et paedagogica, Series altera LXXXII, Lund, Bloms Boktryckeri Ab.

Miller, A. (1990a): For Your Own Good: Hidden Cruelty in Child Rearing and the Roots of Violence, New York, The Noonday Press.

_ (1990b): Banished Knowledge, New York, Double Day Press.

Molinié, G. (1998): Sémiostyslistique, Paris, Presses universitaires de France.

Nord, C. (1997): Translating as a Purposeful Activity. Functionalist Approaches Explained, Manchester, St Jerome Publishing.

Perrot, J. (1993): Actes du Xe Congrès de l'International Research Society for children's literature, Nancy, Presses Universitaires de Nancy.

Peytard, J. (1995) : Mikhaïl Bakhtine. Dialogisme et analyse du discours, Paris, Bertrand-Lacoste.

Reiss, K. and H. J. Vermeer (1984): Grundlegung einer allgemeinen Translationstheorie, Tübingen, Max Niemeyer Verlag.

Shavit, Z. (1986): Poetics of Children Literature, Athens, University of Georgia Press.

Venuti, L. (2000): The Translation Studies Reader, London/New York, Routledge.

\section{REMERCIEMENTS}

Je tiens à remercier Jeanne Dancette qui, par ses corrections et ses remarques toujours pertinentes, a beaucoup aidé à la révision de ce texte.

Percebois, J. (2002) : Terminologie anglais-français du commerce international: théories, politiques, accords et institutions, Paris, Economica, $296 \mathrm{p}$.

L'auteur, maitre de conférences d'anglais à la Faculté des sciences économiques de l'Université de Montpellier, a une longue expérience de l'enseignement de l'anglais économique. Son ouvrage "s'adresse à tous les étudiants dont le cursus inclut des références au commerce international, en particulier les [sic] étudiants des Facultés des Sciences Économiques, AES, IEP, Classes Préparatoires, Écoles supérieures de Commerce et IUT». Il s'inscrit dans le sillage des ouvrages précédents de l'auteur, dont il reprend la formule: L'anglais de la microéconomie et L'anglais de la macroéconomie, publiés chez le même éditeur.

Comment ce livre, présenté comme une terminologie du commerce international, se positionne-t-il par rapport aux nombreux et excellents dictionnaires et manuels d'économie bilingue?

Disons tout d'abord qu'il ne s'agit pas d'un dictionnaire, car il n'est pas organisé en termes. Il ne s'agit pas non plus d'un manuel d'anglais de spécialité, car il ne présente pas une étude de la langue du commerce international. Par sa facture, il ressemble plus à un cours d'économie puisqu'il est structuré autour des grands thèmes classiques du commerce international, comme l'annoncent tant le sous-titre; théories, politiques, accords et institutions, que la table des matières.

Ainsi, les deux premiers chapitres ont pour thèmes les théories consacrées au commerce international. Le troisième chapitre examine le libre-échange et le protectionnisme, puis aborde la question des barrières tarifaires et non tarifaires. Les accords internationaux et les institutions qui les régissent font l'objet du chapitre 4 . Le chapitre 5 traite des blocs 
régionaux et le chapitre 6 est consacré au commerce Nord-Sud. Enfin, le chapitre 7 évoque les liens entre l'agriculture, le commerce et l'environnement.

Ces thèmes forment l'ossature de l'ouvrage. Mais l'originalité du livre réside ailleurs: la matière est traitée dans une approche bilingue, en partant des écrits des économistes de langue anglaise. Les très nombreuses citations qui font la trame du livre sont présentées avec leur traduction française en vis-à-vis, comme le montre la toute première page du livre:

Qu'est-ce que le commerce international?

Selon Paul Krugman et Maurice Obstfeld (1997),

The economics of the international economy can be subdivided into two broad subfields.
La science économique traitant de l'économie internationale peut se diviser en deux grands sous-domaines.

Ces deux sous-domaines sont « international trade analysis» et «international monetary analysis».

International trade analysis focuses primarily on the real transactions in the international economy, that is, on those transactions that involve a physical movement of goods or a tangible commitment of economic resources.

Tandis que

International monetary analysis focuses on the monetary side of the international economy, that is, on financial transactions such as foreign purchases of US dollars.
L'analyse du commerce international se concentre essentiellement sur les transactions réelles de l'économie internationale, c'est-àdire, les transactions qui impliquent un déplacement matériel de biens ou un engagement tangible de ressources économiques.

L'analyse monétaire internationale se concentre sur l'aspect monétaire de l'économie internationale, c'est-à-dire sur les transactions financières telles que les achats de dollars américains à l'étranger.

Cet extrait de Krugman et Obstfeld - qui, par ailleurs, présente une faible valeur pédagogique en tant qu'entrée en matière (trop court, trop abstrait) - donne le ton. De nombreux autres économistes anglais et américains seront cités, tels P. Brenton, H. Scott et P. Sinclair; R.Gilpin; P. Kenen; J. Piggott et M. Cook; W. Walther, insérés dans de très brèves phrases introductives de l'auteur.

Les citations sont entrecoupées de notes terminologiques. Ainsi on aura, à propos de l'occurrence unfair competition (page 9):
fair competition
concurrence loyale
unfair competition
concurrence déloyale

De nombreuses collocations sont données; par exemple, à propos de patents (page 41):
to apply for a patent
déposer une demande de brevet
to take out a patent on sth
faire breveter qch

Donc, l'ouvrage est conçu comme un abrégé d'économie en deux langues. C'est en cela que l'on peut dire qu'il combine «ressources terminologiques et intérêt documentaire». Il se lit dans l'ordre thématique des chapitres (et les notes de vocabulaire sont là pour l'apprentissage de l'anglais). Mais il autorise aussi une recherche par termes au moyen de l'index anglais ou français. On déplorera toutefois que les index, qui contiennent moins de 200 entrées, sont tout à fait insuffisants pour constituer une «terminologie du commerce international». De nombreux termes manquent (clearing agrément, FOB, structural adjustment); de plus, certains termes, pourtant identifiés par l'auteur comme importants puisqu'ils sont en italiques, n'y figurent pas (inflationary/deflationary gap est absent de l'index anglais, et le terme écart est complètement absent de l'index français). 
Pour apprécier la valeur de ce livre et ses qualités pédagogiques, on ne peut s'empêcher de le comparer à d'autres ouvrages. La concurrence est vive dans le domaine économique, couvert par d'excellents ouvrages de référence. Notons, parmi beaucoup d'autres, le Dictionnaire commercial de l'Académie des sciences commerciales, le Dictionnaire de la comptabilité et des sciences de la gestion de P. Ménard, le Lexique d'économie, de Silem et Albertini ou le Dictionnaire économique et financier de Bernard et Colli, et dans une optique très pédagogique, le Dictionnaire d'apprentissage du français des affaires, de Binon et al.

L'ouvrage de J. Percebois ressemble plus en fait à l'excellent livre de F. Garrec, Commerce international et finance - International Trade and Finance (Economica,1991), qui met en contexte bilingue des textes traitant des grandes affaires économiques du jour, mais qui a déjà perdu un peu de son actualité, ou à celui de F. Renversez et al., Économie, dictionnaire encyclopédique (Dalloz, 1998).

Parmi les points forts, nous soulignons la formule originale qui présente en bilingue des textes d'auteurs (là où l'on trouve souvent des articles de journaux); mais nous déplorons le choix des extraits souvent très rébarbatifs car hors contexte et trop courts pour que le lecteur saisisse le fil conducteur. Ce problème, auquel s'ajoute la présentation non linaire des paragraphes, sur deux colonnes (anglais et français) et entrecoupés de notes terminologiques, rend la lecture extrêmement laborieuse. Par contre, les extraits sur les politiques, accords et institutions (moins théoriques) conviennent mieux à la formule du texte en traduction et sont tout à fait intéressants tant sur le plan du contenu que pour les problèmes de langue qu'ils illustrent.

Sur la forme, nous signalerons que l'on aurait pu accorder plus de soin à la traduction (parfois discutable ou trop littérale) et à la correction typographique, puisque de nombreuses fautes de frappe et de coupures de mots anglais ont échappé à la vigilance des réviseurs.

Pour conclure, le livre de J. Percebois mérite de s'inscrire dans la panoplie des ouvrages d'initiation à l'anglais économique par le texte. Toutefois, sa qualité pédagogique aurait pu être bien meilleure si la présentation avait été plus soignée. Du fait de son approche intéressante et originale, l'auteur aurait pu se tailler une place à part parmi les autres ouvrages couvrant ce domaine du commerce international où les besoins des apprenants sont si grands.

JeAnne E. Dancette

ETI, Genève, Suisse

Rouleau, M. (2001): Initiation à la traduction générale. Du mot au texte, Brossard, Linguatech, $241 \mathrm{p}$.

La publication, en 1958, de la Stylistique comparée du français et de l'anglais de Vinay et Darbelnet, œuvre pionnière, montrait la voie à l'enseignement universitaire de la traduction. Depuis, beaucoup d'auteurs, traducteurs ou traductologues, ont suivi le mouvement. On ne compte plus les publications d'ouvrages, rédigés dans la plupart des grandes langues de communication, visant à former des traducteurs, à les initier à l'art et à la pratique d'une activité attestée depuis la plus haute Antiquité. Depuis une vingtaine d'années, l'évolution vers la spécialisation du traducteur aidant, on assiste plus fréquemment à la parution d'ouvrages spécialisés (en commerce, finances et économie, publicité, droit, médecine, technique, etc.), voire théoriques, qu'à celle de manuels de formation à la traduction dite "générale».

C'est pourtant par là, avant de songer à se spécialiser, que le traducteur fera son apprentissage, acquerra les bases de son futur métier et prendra les bonnes habitudes qui lui serviront sa carrière durant. On ne le répètera jamais assez: avant de savoir traduire, il faut savoir écrire, et même bien écrire, préalable obligé à tout projet d'apprentissage de la traduction. Maurice Rouleau, auteur du premier (?) manuel pratique d'initiation à la traduction publié 